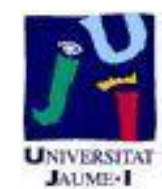

Título artículo / Títol article:

Autores / Autors:

Campos Sancho,

Beatriz ; Cordero,

Alicia; Torregrosa, Juan

R. ; Vindel Cañas, María

Purificacion

Revista:

International Journal of

Computer Mathematics

Versión / Versió:

Postprint

Cita bibliográfica / Cita

bibliogràfica (ISO 690):

CAMPOS, B., et al. Dynamics

of the family of $c$-iterative methods. International Journal

of Computer Mathematics, 2014, no ahead-of-print, p. 1-

11.

url Repositori UJI:

http://hdl.handle.net/10234/13

2269 


\title{
Dynamics of the family of c-iterative methods*
}

\author{
${ }^{1}$ B. Campos, ${ }^{2}$ Alicia Cordero, ${ }^{2}$ Juan R. Torregrosa and ${ }^{1} \mathrm{P}$. Vindel \\ ${ }^{1}$ Instituto de Matemáticas y Aplicaciones de Castellón. Universitat Jaume I. \\ ${ }^{2}$ Instituto de Matemática Multidisciplinar. Universitat Politécnica de Valencia. \\ campos@uji.es, acordero@mat.upv.es, jrtorre@mat.upv.es, vindel@uji.es
}

\begin{abstract}
In this paper, the dynamics of the family of c-iterative methods for solving nonlinear equations are studied on quadratic polynomials. A singular parameter space is presented to show the complexity of the family. The analysis of the parameter space allows us to find elements of the family that have bad convergence properties and also other ones with very stable behavior. These schemes correspond to values of $c$ in different small regions of the parameter space.
\end{abstract}

\section{Introduction}

The application of iterative methods for solving a nonlinear equation $f(z)=0$ with $f: \mathbb{C} \rightarrow \mathbb{C}$, gives rise to rational functions whose dynamics are not well-known. From the numerical point of view, the dynamical properties of the rational function associated with an iterative method give us important information about its stability and reliability. In $[3,6,8,9,10,14,17,20,22]$, the authors study the dynamics of different iterative families: Chebyshev-Halley, King, ...

In most of these studies interesting dynamical planes have been obtained, including some periodical behaviors and other anomalies. However, in contrast to dynamical planes, the parameter planes associated to a family of methods allow us to understand the behavior of the different members of the family of methods, helping us in the selection of a particular one. This has been recently showed in [7], [9] and [10].

The two-parametric third-order iterative root-finding methods

$$
z_{n+1}=z_{n}-\left(1+\frac{1}{2} \frac{L_{f}\left(z_{n}\right)}{1-\alpha L_{f}\left(z_{n}\right)}+c L_{f}\left(z_{n}\right)^{2}\right) \frac{f\left(z_{n}\right)}{f^{\prime}\left(z_{n}\right)}
$$

where

$$
L_{f}(z)=\frac{f(z) f^{\prime \prime}(z)}{\left(f^{\prime}(z)\right)^{2}}
$$

and $\alpha$ and $c$ are complex parameters, include different known families and classical schemes as Chebyshev, Halley, super-Halley or Newton.

*Supported by Ministerio de Ciencia y Tecnología MTM2011-28636-C02-02. The first and fourth authors were also partially supported by P11B2011-30 (Universitat Jaume I), the second and third authors were also partially supported by Vicerrectorado de Investigación, Universitat Politècnica de València PAID-06-2010-2285. 
In fact, for $c=0$ the Chebyshev-Halley family is obtained. From a numerical point of view, it has been widely studied (see, for instance, [15] and [16]) and the authors have started its dynamical study in [10] and [11] and [12].

If $\alpha=0$ and $c$ is different from zero, the so-called $c$-iterative methods are obtained (see [2]). This family has been scarcely studied, both from a numerical and dynamical point of view. So, the main goal of this paper is to deep in the dynamical analysis of this class of methods, whose fixed point operator is:

$$
M_{f, c}(z)=z-\left(1+\frac{1}{2} L_{f}(z)+c L_{f}(z)^{2}\right) \frac{f(z)}{f^{\prime}(z)} .
$$

In [1], a Scaling theorem is proved for this family. So, the behavior of this class of methods on quadratic polynomials can be reduced to the study of these schemes applied on $p(z)=z^{2}+a$. For this polynomial, the operator (1) is associated with the rational function:

$$
M_{p}(z, a, c)=\frac{-1}{8 z^{5}}\left(a^{3} c+a^{2}(1+3 c) z^{2}+3 a(2+c) z^{4}+(-3+c) z^{6}\right),
$$

depending on two complex parameters: $a$ and $c$.

The parameter $a$ can be obviated by considering the conjugacy map (see [5])

$$
h(z)=\frac{z-i \sqrt{a}}{z+i \sqrt{a}}
$$

with the following properties:

$$
h(\infty)=1, \quad h(i \sqrt{a})=0, \quad h(-i \sqrt{a})=\infty .
$$

Then, the operator $M_{p}(z, a, c)$ is conjugated to:

$$
O_{p}(z, c)=\left(h \circ M_{p} \circ h^{-1}\right)(z, c)=z^{3} \frac{-4 c+(1+z)^{2}(2+z)}{1+4 z+5 z^{2}+2(1-2 c) z^{3}} .
$$

Now, we recall some dynamical concepts of complex dynamics (see [4]) that we use in this paper. Given a rational function $R: \hat{\mathbb{C}} \rightarrow \hat{\mathbb{C}}$, where $\hat{\mathbb{C}}$ is the Riemann sphere, the orbit of a point $z_{0} \in \hat{\mathbb{C}}$ is defined as:

$$
\left\{z_{0}, R\left(z_{0}\right), R^{2}\left(z_{0}\right), \ldots, R^{n}\left(z_{0}\right), \ldots\right\}
$$

We analyze the phase plane of the map $R$ by classifying the starting points from the asymptotic behavior of their orbits. A $z_{0} \in \hat{\mathbb{C}}$ is called a fixed point if $R\left(z_{0}\right)=z_{0}$. A periodic point $z_{0}$ of period $p>1$ is a point such that $R^{p}\left(z_{0}\right)=z_{0}$ and $R^{k}\left(z_{0}\right) \neq z_{0}$, for $k<p$. A pre-periodic point is a point $z_{0}$ that is not periodic but there exists a $k>0$ such that $R^{k}\left(z_{0}\right)$ is periodic. A point $z_{0}$ is a critical point of the rational map $R$ if $R$ fails to be injective in any neighborhood of $z_{0}$. Moreover, a fixed point $z_{0}$ is called attractor if $\left|R^{\prime}\left(z_{0}\right)\right|<1$, superattractor if $\left|R^{\prime}\left(z_{0}\right)\right|=0$, repulsor if $\left|R^{\prime}\left(z_{0}\right)\right|>1$ and parabolic if $\left|R^{\prime}\left(z_{0}\right)\right|=1$. The fixed points different from the roots of the polynomial $p(z)$ are called strange fixed points.

The basin of attraction of an attractor $\alpha$ is defined as:

$$
\mathcal{A}(\alpha)=\left\{z_{0} \in \hat{\mathbb{C}}: R^{n}\left(z_{0}\right) \rightarrow \alpha, n \rightarrow \infty\right\} .
$$


The Fatou set of the rational function $R, \mathcal{F}(R)$, is the set of points $z \in \hat{\mathbb{C}}$ whose orbits tend to an attractor (fixed point, periodic orbit or infinity). Its complement in $\hat{\mathbb{C}}$ is the Julia set, $\mathcal{J}(R)$. That means that the basin of attraction of any fixed point belongs to the Fatou set and the boundaries of these basins of attraction belong to the Julia set.

The rest of the paper is organized as follows: in Section 2 we analyze the fixed and critical points of the operator $O_{p}(z, c)$ and in Section 3 we show the parameter space and study the stability of the fixed points. In Section 4, the dynamics of some conflictive methods of the family is presented. We finish the work with some remarks and conclusions.

\section{Study of the fixed and critical points}

We study the dynamics of the operator $(2)$ in function of the parameter $c$. Firstly, we calculate the fixed and the critical points of $O_{p}(z, c)$. As we see in the following, the number and stability of fixed and critical points depend on the value of parameter $c$.

It is known that fixed points satisfy $O_{p}(z, c)=z$. The solutions are $z=0$ and $z=\infty$, associated to the roots of $p(z)$, and the strange fixed points:

$$
\begin{aligned}
z & =1, \text { if } c \neq 3, \\
z_{1}(c) & =\frac{1}{4}(-5-\sqrt{1-16 c}+\sqrt{2} \sqrt{5-8 c+5 \sqrt{1-16 c}}), \\
z_{2}(c) & =\frac{1}{4}(-5-\sqrt{1-16 c}-\sqrt{2} \sqrt{5-8 c+5 \sqrt{1-16 c}}), \\
z_{3}(c) & =\frac{1}{4}(-5+\sqrt{1-16 c}-\sqrt{2} \sqrt{5-8 c-5 \sqrt{1-16 c}}), \\
z_{4}(c) & =\frac{1}{4}(-5+\sqrt{1-16 c}+\sqrt{2} \sqrt{5-8 c-5 \sqrt{1-16 c}}) .
\end{aligned}
$$

It is easy to show that $z_{1}=\frac{1}{z_{2}}$ and $z_{3}=\frac{1}{z_{4}}$ and the relations established in the following lemma.

Lemma 1 The number of strange fixed points of $O_{p}(z, c)$ is five, except in the following cases:

i) If $c=0$, then $z_{3}=z_{4}=-1$;

i) If $c=3$, there exist four imaginary strange fixed points ;

ii) If $c=-5$, then $z_{3}=z_{4}=1$;

iii) If $c=\frac{1}{16}$, then $z_{1}=z_{4}=-\frac{1}{2}$ and $z_{2}=z_{3}=-2$.

We show these behaviors in the diagrams of Figure 1, for real values of $c$. We can also observe the real values of the strange fixed points $z_{i}(c), i=1,2,3,4$ and the free critical points $c p_{i}(c), i=1,2$. In this figure, the fixed points are represented in colors blue, red, magenta and green and free critical points are presented in dashed and dotted lines, respectively.

Critical points can be obtained by solving the equation $O_{p}^{\prime}(z, c)=0$. The roots of this equation are

$$
z=0, \quad z=\infty
$$




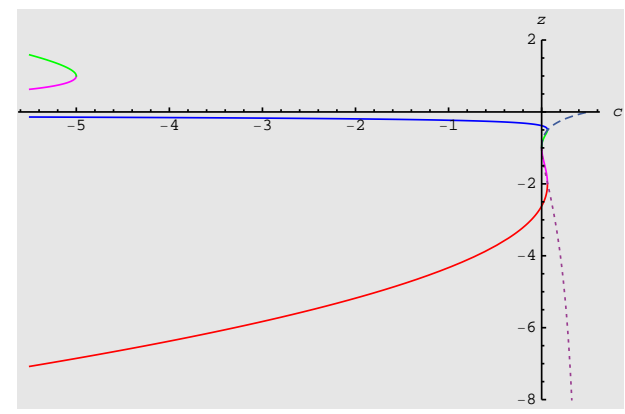

(a) Strange fixed points

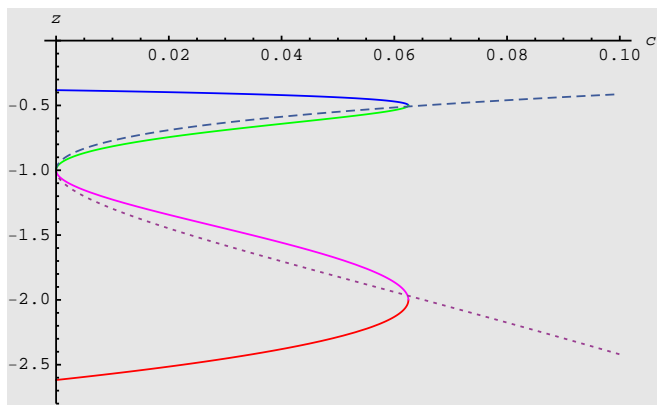

(b) A detail

Figure 1: Relationship between strange fixed and critical points.

and the free critical points are

$$
\begin{aligned}
z & =-1 \\
c p_{1}(c) & =\frac{1}{6 c-3}(4 c-2 \sqrt{5} \sqrt{-c(c-3)}+3), \\
c p_{2}(c) & =\frac{1}{6 c-3}(4 c+2 \sqrt{5} \sqrt{-c(c-3)}+3) .
\end{aligned}
$$

For $c=\frac{1}{2}, c p_{1}(c)$ tends to $0, c p_{2}(c)$ tends to $\infty$ and the only free critical point is $z=-1$.

The critical points $c p_{1}(c)$ and $c p_{2}(c)$ satisfy $c p_{1}(c)=\frac{1}{c p_{2}(c)}$. The critical point $z=-1$ is a preperiodical point of $z=1, O_{p}(-1, c)=1$.

The number of critical points depends on the value of the parameter $c$, summarized in the following lemma.

Lemma 2 The number of free critical points of $O_{p}(z, c)$ is three, except in the following cases:

i) For $c=0, O_{p}^{\prime}(z, 0)=\frac{6 z^{2}(1+z)^{2}}{(1+2 z)^{2}}$ and $c p_{1}=c p_{2}=-1$ is the unique free critical point.

ii) For $c=\frac{1}{2}, O_{p}^{\prime}\left(z, \frac{1}{2}\right)=20 z^{3} \frac{(z+1)^{4}}{\left(5 z^{2}+4 z+1\right)^{2}}$, and $z=-1$ is the only free critical point.

iii) For $c=\frac{1}{27}, O_{p}^{\prime}\left(z, \frac{1}{27}\right)=270 \frac{z^{2}(z+1)^{4}(3 z+5)}{(2 z+3)^{2}(5 z+3)^{3}}$. Then, we have only two free critical points: $z=-1$ and $z=-\frac{5}{3}$.

iv) For $c=3, O_{p}^{\prime}(z, 3)=-30 z^{2} \frac{(1+z)^{4}}{\left(1+5 z+10 z^{2}\right)^{2}}$. Then, only $z=-1$ is a free critical point.

Let us note that in case $c=3, c p_{1}(3)=c p_{2}(3)=1$, that is a strange fixed point, but they are not critical points due to the simplification of the rational function.

\section{Study of the parameter space}

The dynamical behavior of the operator (2) depends on the value of the parameter $c$ and it is obtained by applying the operator on one of the critical points and coloring the initial conditions depending on whether this critical goes to the basin of attraction of 0 or $\infty$ (dark 
color) or to another unknown basin of attraction (white color). Moreover, each point of the parameter plane is associated with a complex value of $c$, i.e., with an element of the family (2). Every value of $c$ belonging to the same connected component of the parameter space gives rise to subsets of schemes of the family (2) with equivalent dynamical behavior (see [7] for more information about the codes to generate parameter and dynamical planes).

Let us notice that the free critical point $z=-1$ is a preimage of the strange fixed point $z=1$, so there is no sense in analyzing its associated parameter space. Nevertheless, we know that $c p_{1}(c)=\frac{1}{c p_{2}(c)}$. Then, they have the same parameter space that can be seen in Figure 2 .

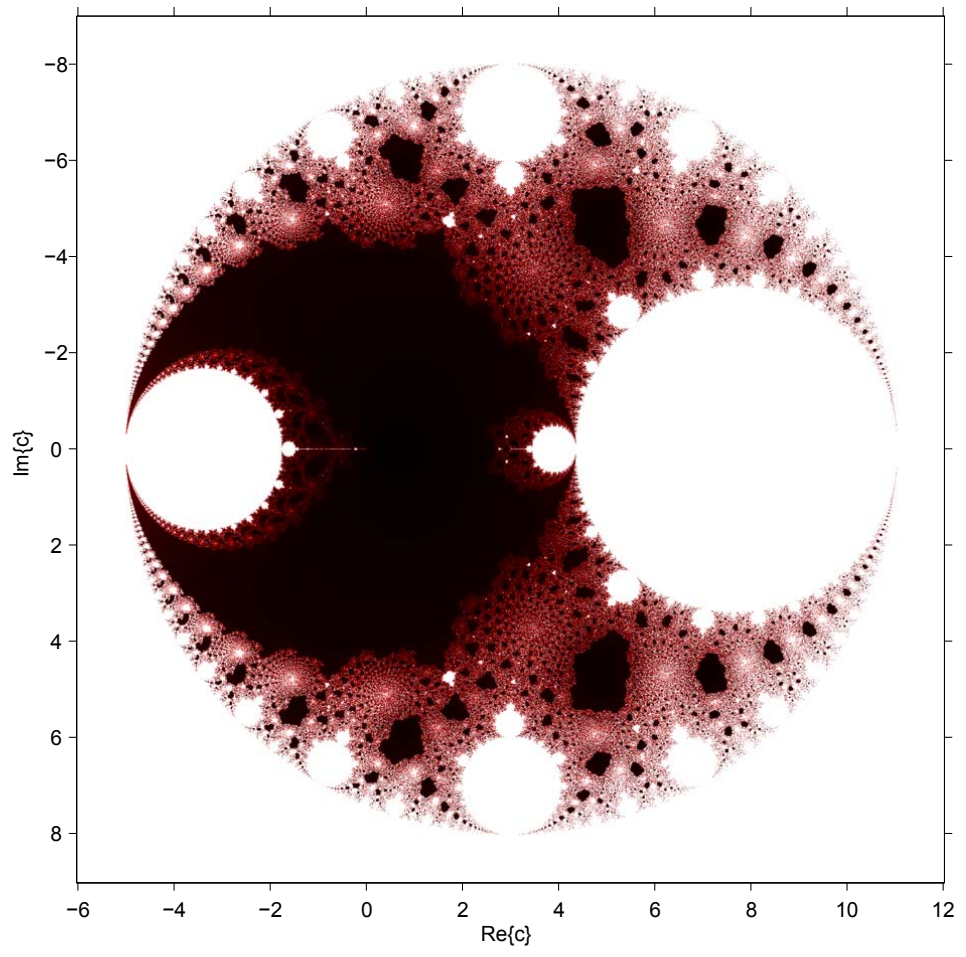

Figure 2: Parameter plane.

As some of the white regions of this parametric plane are related to the values of parameter $c$ for which some of the strange fixed points are attractive, we study the stability regions of those points.

\subsection{Stability of the fixed points}

To study the stability of the fixed points we need the derivative of the operator (2):

$$
O_{p}^{\prime}(z, c)=-2 z^{2}(1+z)^{4} \frac{-3(1+z)^{2}+2 c\left(3-4 z+3 z^{2}\right)}{\left(1+4 z+5 z^{2}+2(1-2 c) z^{3}\right)^{2}} .
$$

From (7) we obtain that the origin and $\infty$ are always superattractive fixed points, but the stability of the other fixed points changes depending on the values of the parameter $c$. The stability function of the fixed point $z=1$ is

$$
\left|O_{p}^{\prime}(1, c)\right|=\left|\frac{8}{3-c}\right|
$$


This function allows us to state this result:

Lemma 3 The stability of the strange fixed point $z=1$ satisfies the following statements:

i) If $|c-3|>8$ then $z=1$ is an attractor.

ii) For $|c-3|=8,\left|O_{p}^{\prime}(1)\right|=1$, so $z=1$ is a parabolic point in this circle.

iii) Finally, $z=1$ is a repulsive fixed point for the disk $|c-3|<8$.

Let us remark that $z=1$ is attractive in the white region of the parameter space (see Figure 2 ) outside of the circle centered at $(3,0)$ and radius $r=8$. We also observe that $z=1$ is not a fixed point of the operator for $c=3$, because:

$$
O_{p}(z, 3)=-z^{3} \frac{\left(5 z+z^{2}+10\right)}{\left(5 z+10 z^{2}+1\right)} \Rightarrow O_{p}(1,3)=-1 .
$$

On the other hand, we can see in Figures 3 and $5 \mathrm{~b}$ that $z_{1}(c)$ and $z_{2}(c)$ are always repulsive fixed points; $z_{3}(c)$ and $z_{4}(c)$ are attractive in a small interval. In fact, the intersection points between the stability function of $z_{3}(c)$ and $z_{4}(c)$ with $z=1$ are $c=-5$ and $c=c_{0}=\frac{-109-11 \sqrt{113}}{128} \approx-1.765090655781884$. For $c$ real, the stability functions of the strange fixed points $z_{1}(c), z_{2}(c), z_{3}(c)$ and $z_{4}(c)$, satisfy the following result.

Lemma 4 Let us consider c real. The fixed points $z_{i}(c), i=1,2,3,4$ satisfy the following statements of stability:

i) If $-5<c<c_{0}$, then $z_{3}$ and $z_{4}$ are attractive fixed points.

ii) For $c=-5$, then $z_{3}=z_{4}=1$ and it is a parabolic point.

iii) For $c=c_{0}, O_{p}^{\prime}\left(z_{3}, c_{0}\right)=O_{p}^{\prime}\left(z_{4}, c_{0}\right)=-1$ and they are parabolic points, where $z_{3} \approx$ $0.101884-0.994796 i, z_{4} \approx 0.101884+0.994796 i$.

iv) $z_{3}$ and $z_{4}$ are repulsive fixed points for any other real value of $c$.

v) $z_{1}$ and $z_{2}$ are repulsive for any value of $c$.

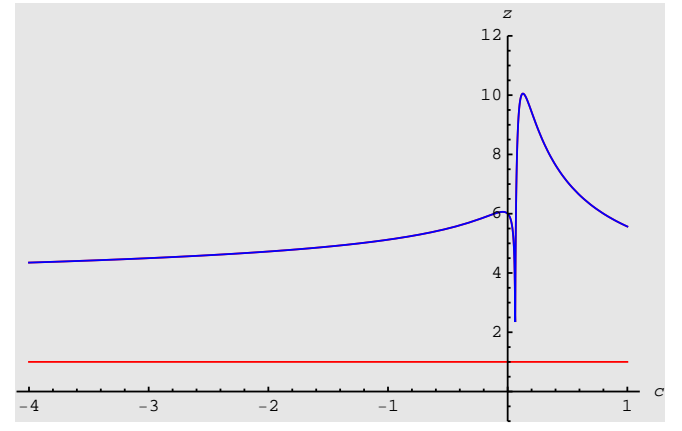

(a) $\left|O_{p}^{\prime}\left(z_{i}(c)\right)\right|, \quad i=1,2$.

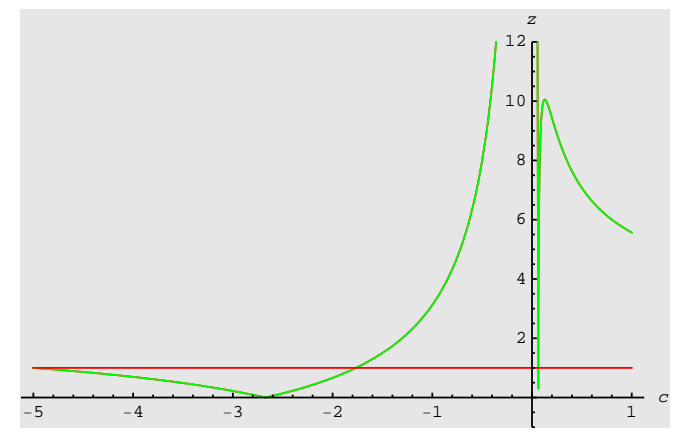

(b) $\left|O_{p}^{\prime}\left(z_{i}(c)\right)\right|, \quad i=3,4$.

Figure 3: Stability functions for strange fixed points $z_{i}(c), i=1,2,3,4$.

This behavior can also be observed in the parameter plane (see Figure 2) where, as we prove in the following, where the white disk on the left corresponds to the complex values of $\mathrm{c}$ for 
which $z_{3}$ and $z_{4}$ are attractive. Let us note that this white region is not exactly a disk but it is embedded in a corona. Moreover, we see that $z_{1}$ and $z_{2}$ are repulsive for any complex value of $c$.

Lemma 5 Let $D$ be the region of the complex plane where the fixed points $z_{3}$ and $z_{4}$ are attractive. Then,

$$
D_{1}:=\{c:|c-m|<1.5\} \subset D \subset D_{2}:=\{c:|c-m|<1.7\}
$$

with $m=\frac{-5+c_{0}}{2}$ and $c_{0}=\frac{-109-11 \sqrt{113}}{128}$.

Proof. By definition of $D,\left|O_{p}^{\prime}\left(z_{3}, c\right)\right|=\left|O_{p}^{\prime}\left(z_{4}, c\right)\right|<1$ for all $c \in D$. Based on the study of real values of $c$, we consider the circle $\hat{D}$ centered in $m=\frac{-5+c_{0}}{2}$ and radius $r=\frac{5+c_{0}}{2} \cong 1.61745$. In Figure $4 \mathrm{~b}$ we show the stability functions of $z_{3}(c)$ and $z_{4}(c)$ evaluated in $\partial D_{2}$ (blue line), in $\partial \hat{D}$ (red line) and in $\partial D_{1}$ (green line). We can observe that $\left|O_{p}^{\prime}\left(z_{3}\right)\right|=\left|O_{p}^{\prime}\left(z_{4}\right)\right| \leq 1$ for the points on $\partial \hat{D}$; these functions verify $\left|O_{p}^{\prime}\left(z_{3}\right)\right|=\left|O_{p}^{\prime}\left(z_{4}\right)\right|<1$ on the points of $C_{1}=\partial D_{1}$ and they verify that $\left|O_{p}^{\prime}\left(z_{3}\right)\right|=\left|O_{p}^{\prime}\left(z_{4}\right)\right|>1$ on $C_{2}=\partial D_{2}$.

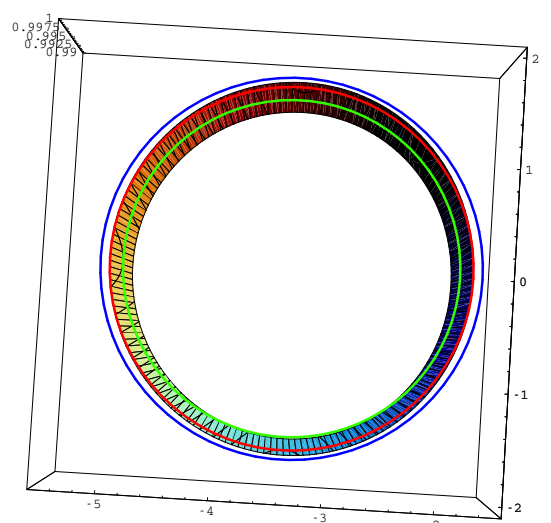

(a) Stability functions $\left|O_{p}^{\prime}\left(z_{3}\right)\right|=$ $\left|O_{p}^{\prime}\left(z_{4}\right)\right|$ and the circles

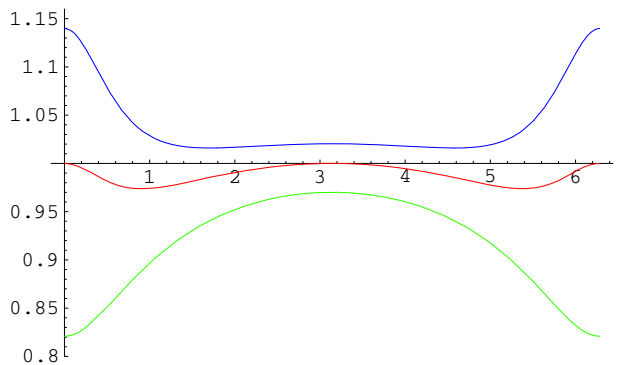

(b) $\left|O_{p}^{\prime}\left(z_{3}\right)\right|=\left|O_{p}^{\prime}\left(z_{4}\right)\right|$ on the circles

Figure 4: Study of $\left|O_{p}^{\prime}\left(z_{3}\right)\right|$ and $\left|O_{p}^{\prime}\left(z_{4}\right)\right|$.

So, the boundary of $D$ is not a circle but it is embedded in a corona delimited by the circles $C_{1}$ and $C_{2}$ (Figure 4a). The values $\left|O_{p, c}^{\prime}\left(z_{3}\right)\right|=\left|O_{p, c}^{\prime}\left(z_{4}\right)\right|=1$ correspond to $c=-5$ and $c=c_{0}$.

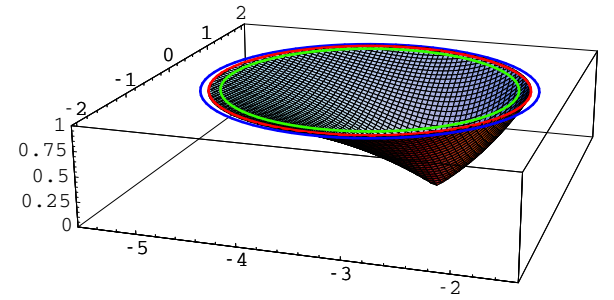

(a) Stability functions $\left|O_{p}^{\prime}\left(z_{3}\right)\right|=\left|O_{p}^{\prime}\left(z_{4}\right)\right|$

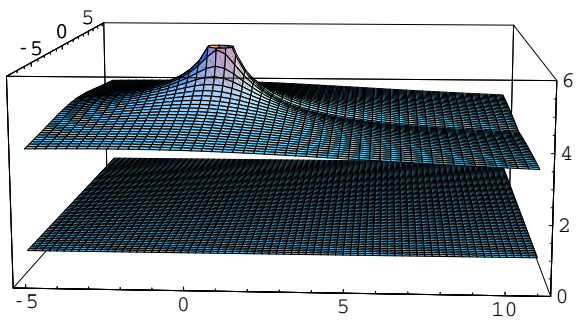

(b) $\left|O_{p}^{\prime}\left(z_{1}\right)\right|$ and $\left|O_{p}^{\prime}\left(z_{2}\right)\right|$

Figure 5: Stability functions. 
On the other hand, the stability functions of the other strange fixed points, $z_{1}$ and $z_{2}$, show that they are repulsive for any value of $c$ (Figure 5b).

\subsection{Dynamical planes}

In this section we study the dynamical planes corresponding to values of $c$ inside different connected components in the parameter space. These dynamical planes have been obtained by using the software included in [7], implemented in Matlab. The colors used also give us important information: orange regions are the basins of attraction of the fixed point 0 ; blue regions correspond to the basin of the infinity and the area of convergence of other strange fixed points are shown in other colors. Moreover, black regions are associated to attractive periodical orbits.

From Lemma 3, we know that for any value $c$ such that $|c-3|>8$, the fixed point $z=1$ is an attractor. So, for such values the dynamical planes have three different basins of attractions, one for each attractive fixed point, $0, \infty$ and 1 . Then, the dynamical planes are similar to those of Figures $6 \mathrm{a}$ and $6 \mathrm{~b}$. Let us observe that the basin of attraction of $z=1$ increases when $c$ moves away from 3 .

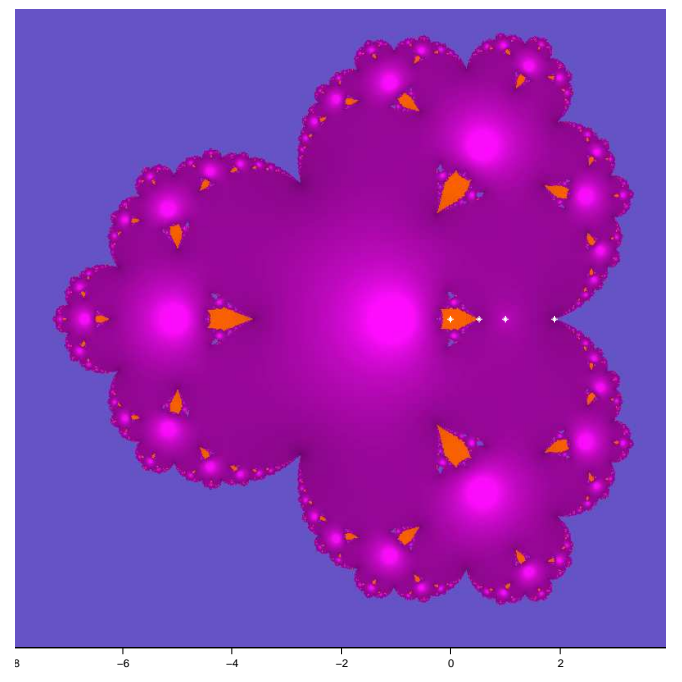

(a) $c=-6$

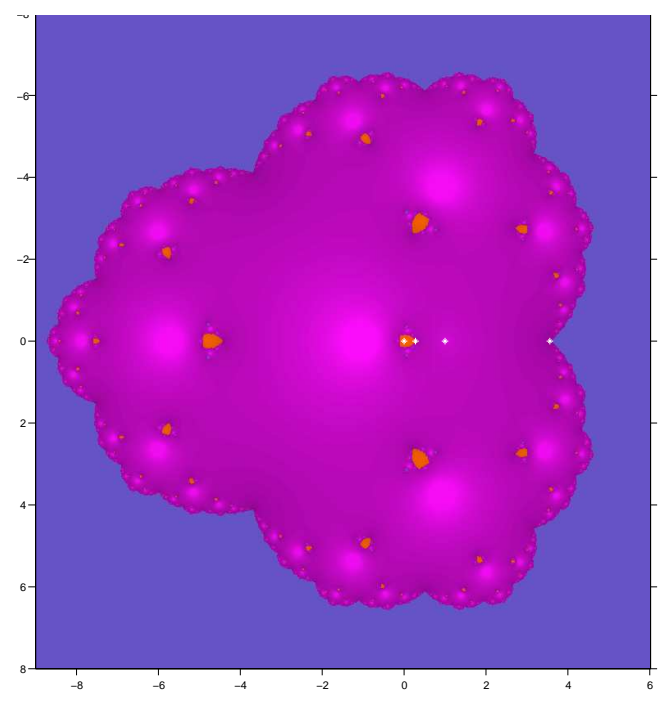

(b) $c=-10$

Figure 6: Dynamical planes where $z=1$ is an attractor.

In the parameter plane (see Figure 2), the circle centered at $c=3$ with radius 8 is the locus of bifurcation of $z=1$. Different types of bifurcations are found on this locus. We also have observed a symmetry respect to the real line. For example, for $c=3+7 i$ and $c=3-7 i$ the fixed point $z=1$ is parabolic and it becomes repulsive when entering in the nearby white disk, and one attractive orbit of period 4 appears (see Figures $7 \mathrm{a}$ and $7 \mathrm{~b}$ ).

As it happens in other parameter spaces, the difference between these bifurcations is that the rotation of the orbits is reversed. Similarly, for $c=7+4 \sqrt{3} i$ and $c=7-4 \sqrt{3} i$, the fixed point $z=1$ bifurcates to orbits of period 3 when entering in the nearby white disk (see Figure $8 \mathrm{a}$ and $8 \mathrm{~b})$.

Let us observe that for bifurcations obtained outside the real line the fixed point $z=1$ becomes repulsive and attractive orbits of period $p$ appears. Nevertheless, when $c$ is moving on the real line we find other type of bifurcations. 


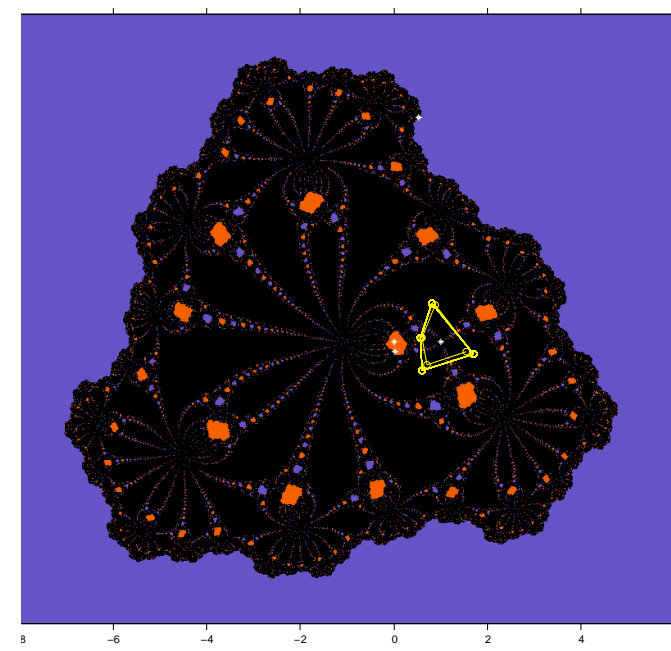

(a) $c=3+7 i$

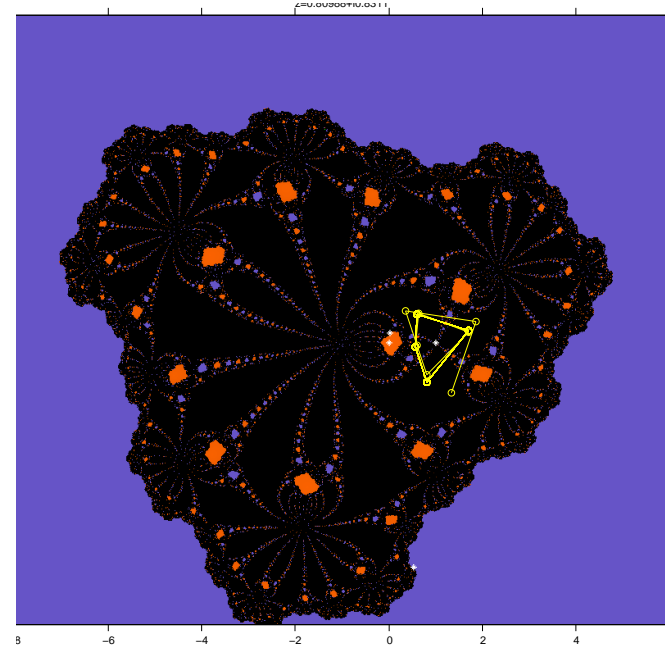

(b) $c=3-7 i$

Figure 7: Dynamical planes for $c=3 \pm 7 i$.

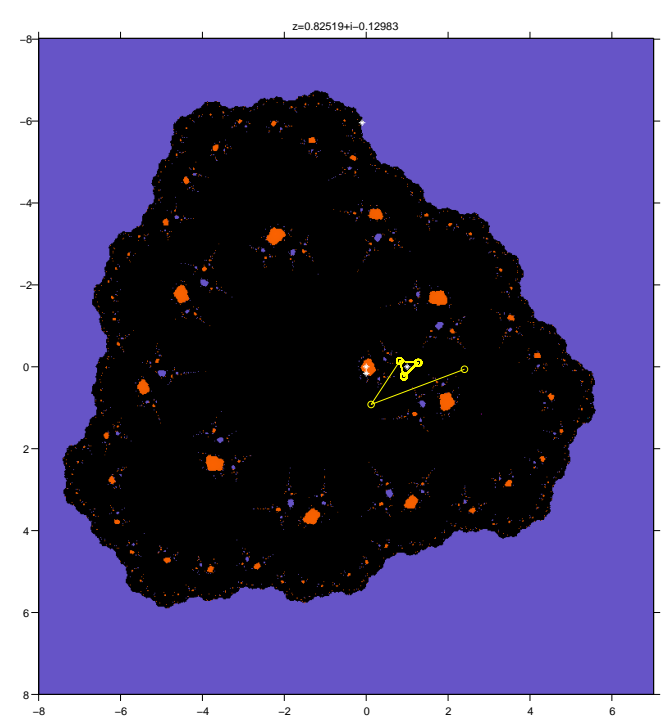

(a) $c=7+4 \sqrt{3} i$

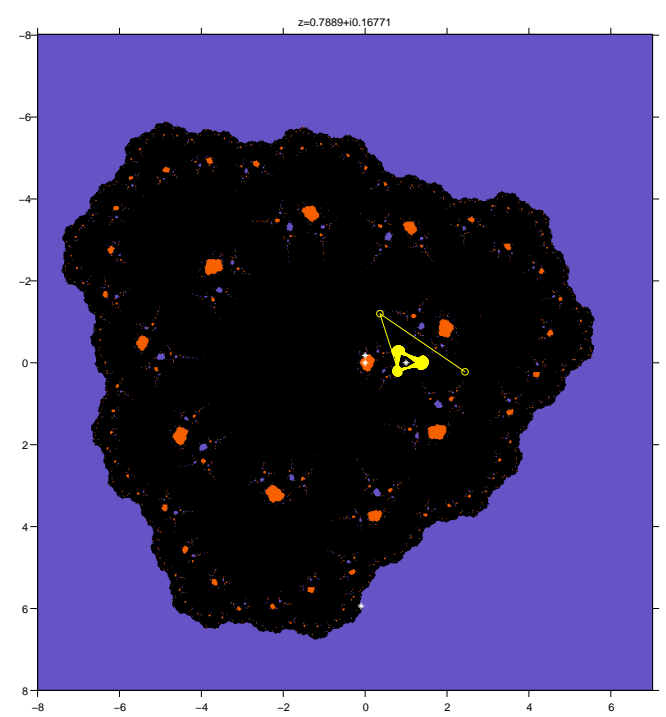

(b) $c=7-4 \sqrt{3} i$

Figure 8: Orbits of period 3.

Let us now study the two big white disks cutting the real line. For $c=-5$, as we have seen before, the fixed point $z=1$ bifurcates to the two fixed points $z_{3}$ and $z_{4}$ and these points are attractive fixed points in the whole white disk $D$ (Lemma 5). So, the dynamical planes for these values of the parameter have four different basins of attraction: $0, \infty, z_{3}$ and $z_{4}$. They are similar to that shown in Figure 9a.

If we increase $c$ moving on the real line, we find that for $c=c_{0}$, two double period bifurcations occur; the points $z_{3}$ and $z_{4}$ become repulsive and two periodic orbits of period 2 appear.

Now, we analyze the white disk on the right; when $c=4$, there is a new bifurcation value 


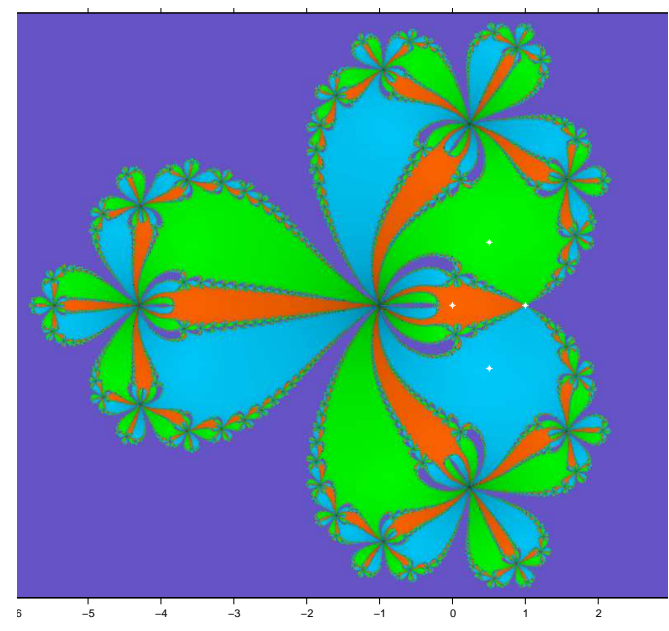

(a) Dynamical plane for $c=-3$

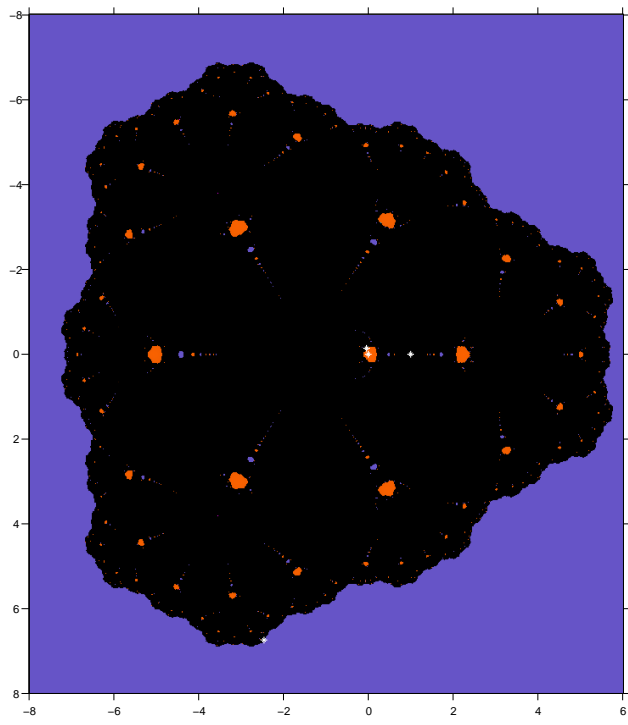

(b) Dynamical plane for $c=11$

Figure 9: Dynamics in $|c-3|<8$.

where this double period orbit becomes repulsive and two attractive orbits of period 2 appear (see Figures 10a and 10b). For $c=11$, a double period bifurcation occurs; the point $z=1$

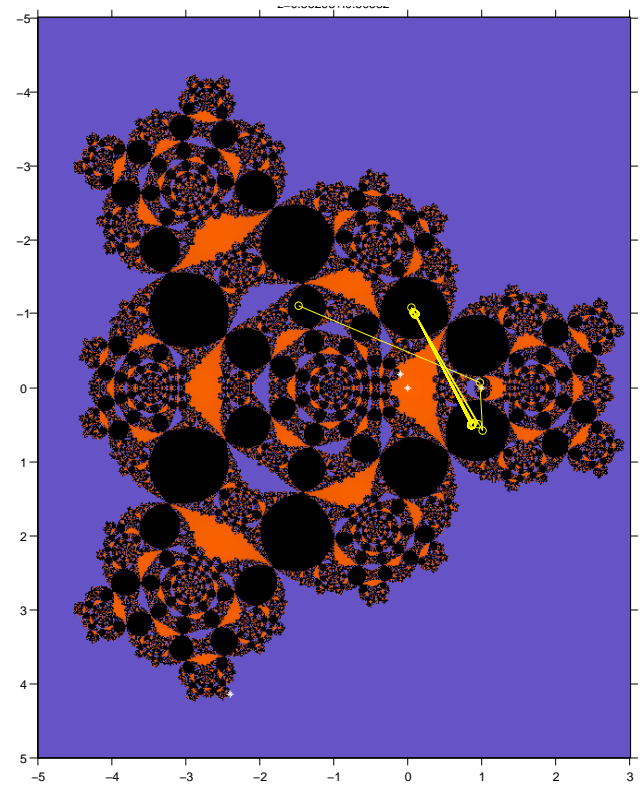

(a) Orbit 1

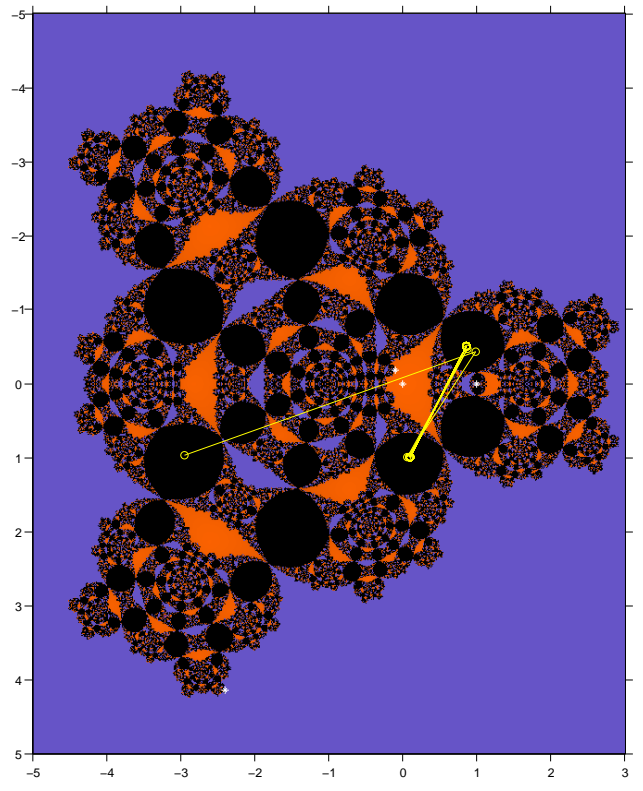

(b) Orbit 2

Figure 10: Dynamical planes for $c=4$.

becomes repulsive and one orbit of period 2 appears (see Figure $9 \mathrm{~b}$ ).

Finally, let us remark that the dark region in the parameter space corresponds to dynamical planes where the only attractive points are 0 and $\infty$, that is, the roots of the polynomial. This is the region with good numerical behavior. The associated dynamical planes for this region are similar to the one of Newton's scheme. 


\section{Conclusions}

The dynamical behavior of the family of c-iterative methods on quadratic polynomials is very rich. It has been proved that there are wide regions with no convergence to the roots, and the existence of periodic orbits of arbitrary period has been showed. On the other hand, there are small regions in the parameter space whose corresponding iterative methods have a good numerical behavior. These are associated to the black areas in the parameter space.

Interesting future studies deals with the dynamical analysis inside and in the boundary of the white bulbs and black areas in the parameter space.

Acknowledgements: The authors would like to thank the anonymous referees for their valuable suggestions and comments.

\section{References}

[1] S. Amat, C. Bermúdez, S. Busquier and S. Plaza, Review of some iterative root-finding methods from a dynamical point of view, Scientia Series A: Mathematical Sciences 10 (2004), pp. 3-35.

[2] S. Amat, S. Busquier and J.M. Gutiérrez, Geometric constructions of iterative functions to solve nonlinear equations, Journal of Computational and Applied Matheatics 157(1) (2003), pp. 197-205.

[3] S. Artidiello, F. Chicharro, A. Cordero and J.R. Torregrosa, Local convergence and dynamical analysis of a new family of optimal fourth-order iterative methods, International Journal of Computer Mathematics doi:10.1080/00207160.2012.748900.

[4] P. Blanchard, Complex Analytic Dynamics on the Riemann Sphere, Bull. of the AMS 11(1) (1984), pp. 85-141.

[5] P. Blanchard, The Dynamics of Newton's Method, Proc. of Symposia in Applied Mathematics 49 (1994), pp. 139-154.

[6] F. Chicharro, A. Cordero, J.M. Gutiérrez and J.R. Torregrosa, Complex dynamics of derivative-free methods for nonlinear equations, Applied Mathematics and Computation doi: $10.1016 /$ j.amc.2012.12.075.

[7] F. Chicharro, A. Cordero and J.R. Torregrosa, Drawing dynamical and parameter planes of iterative families and methods, The Scientific World Journal Volume 2013 Article ID 780153.

[8] C. Chun, M.Y. Lee, B. Neta and J. Džunić, On optimal fourth-order iterative methods free from second derivative and their dynamics, Applied Mathematics and Computation 218 (2012), pp. 6427-6438.

[9] A. Cordero, J. García-Maimó, J.R. Torregrosa, M.P. Vassileva and P. Vindel, Chaos in King's iterative family, Applied Mathematics Letters 26 (2013), pp. 842-848.

[10] A. Cordero, J.R. Torregrosa and P. Vindel. Dynamics of a family of Chebyshev-Halley type methods. Applied Mathematics and Computation 219 (2013), 8568-8583. 
[11] A. Cordero, J.R. Torregrosa and P. Vindel, Period-doubling bifurcations in the family of Chebyshev-Halley type methods, International Journal of Computer Mathematics doi:10.1080/00207160.2012.745518.

[12] A. Cordero, J.R. Torregrosa and P. Vindel. Bulbs of period two in family of ChebyshevHalley type methods on quadratic polynomials. Abstract and Applied Analysis Volume 2013, ID 53691010 pages.

[13] A. Douady and J.H.Hubbard, On the dynamics of polynomials-like mappings, Ann. Sci. Ec. Norm. Sup. (Paris) 18 (1985), 287-343.

[14] J.M. Gutiérrez, M.A. Hernández and N. Romero, Dynamics of a new family of iterative processes for quadratic polynomials, J. of Computational and Applied Mathematics 233 (2010), pp. 2688-2695.

[15] J.M. Gutiérrez and M.A. Hernández, A family of Chebyshev-Halley type methods in Banach spaces, Bulletin of the Australian Mathematical Society 55 (1997), pp. 113-130.

[16] M.A. Hernández and M.A. Salanova, A family of Chebyshev-Halley type methods, International Journal of Computer Mathematics 47 (1-2) (1993), pp. 59-63.

[17] G. Honorato, S. Plaza and N. Romero, Dynamics of a high-order family of iterative methods, Journal of Complexity 27 (2011), pp. 221-229.

[18] C. McMullen, Family of rational maps and iteraive root-finding algorithms, Ann. of Math. 125 (1987), pp. 467-493.

[19] J. Milnor, Dynamics in one complex variable, Princeton University Press, 2006.

[20] B. Neta, M. Scott and C. Chun, Basin attractors for various methods for multiple roots, Applied Mathematics and Computation 218 (2012), pp. 5043-5066.

[21] N. Osada, Chebyshev-Halley methods for analytic functions, Journal of Computational and Applied Mathematics 216(2) (2008), pp. 585-599.

[22] M. Scott, B. Neta and C. Chun, Basin attractors for various methods, Applied Mathematics and Computation 218 (2011), pp. 2584-2599.

[23] J.F. Traub, Iterative methods for resolution of equations, Prentice Hall, NJ 1964.

[24] J.L. Varona. Graphic and numerical comparison between iterative methods. Math. Intelligencer 24(1) (2002), pp. 37-46. 